\title{
Augmented cardiovascular responses to episodes of repetitive compared with isolated respiratory events in preschool children with sleep-disordered breathing
}

\author{
Lisa M. Walter ${ }^{1,2}$, Sarah N. Biggs ${ }^{1,2}$, Lauren C. Nisbet' ${ }^{1}$, Aidan J. Weichard' ${ }^{1}$, Milou Muntinga' ${ }^{1}$, Margot J. Davey ${ }^{1-3}$, \\ Vicki Anderson ${ }^{4}$, Gillian M. Nixon ${ }^{1-3}$ and Rosemary S.C. Horne ${ }^{1,2}$
}

BACKGROUND: Childhood sleep disordered breathing (SDB) presents as isolated respiratory events or episodes of consecutive repetitive events. We hypothesized that the surge in blood pressure (BP) and heart rate (HR) would be greater at the termination of events during episodes of repetitive events than following isolated events.

METHODS: \% change in HR and pulse transit time (PTT; inverse surrogate of BP) were calculated from the last half of an event to: (i) between successive repetitive events; (ii) termination of the last repetitive event; (iii) event termination for isolated events.

RESULTS: $69 \%$ of the children exhibiting both isolated and repetitive events had more repetitive than isolated events. $\% H R$ change between repetitive events (27 $\pm 1 \%$ ) was greater than at event termination for isolated events $(17 \pm 1 \%$; $P<0.001)$. \%PTT change at the termination of the last repetitive event $(-8 \pm 2 \%)$ was greater than at the termination of isolated events $(-2 \pm 2 \% ; P<0.05)$.

CONCLUSION: Episodes of repetitive respiratory events evoke a greater acute cardiovascular response, including surges in BP and HR between events, than do isolated events. Given that the majority of respiratory events in preschool children occur as repetitive episodes, this finding should be taken into account when assessing the impact of respiratory events for a given child.

$S^{\text {lee }}$ eep-disordered breathing (SDB) is a term used to describe both obstructive and central respiratory disturbances during sleep. Obstructive SDB is a common disorder in children especially during the preschool years (1). In children, obstructive events are usually due to adenotonsillar hypertrophy, which can contribute to the complete or partial obstruction of the upper airway by the enlarged lymphoid tissue during sleep when the respiratory muscles of the upper airway relax and can no longer maintain an open airway (2). Obstructive SDB forms a continuum of severity ranging from primary snoring to obstructive sleep apnea (OSA).
SDB has been associated with elevated heart rate (HR) and blood pressure (BP) in children (3-7). In both adults $(8,9)$ and children $(10,11)$ with SDB, obstructive apneas and hypopneas are associated with a decrease in HR and BP during the event followed by a significant surge in HR and BP at event termination. In addition to obstructive respiratory events, adults, children, and neonates can exhibit central apneas during sleep, where there is no, or reduced, brain stem motor output. Central apneas have been described in healthy children during sleep and are known to decrease with age $(12,13)$. A similar cardiovascular response is also associated with central apneas in children with SDB (14). In addition, respiratory events may be associated with hypoxia and arousal, both of which have cardiovascular sequelae, increasing $\mathrm{BP}, \mathrm{HR}$, and sympathetic activity (15-18). It is thought that the cardiovascular morbidity associated with SDB may in part be a consequence of the repetitive swings in BP and HR associated with respiratory events (for review see ref. (19)).

During sleep, adults and children with SDB have both isolated respiratory events and episodes of repetitive respiratory events, which can include a combination of both obstructive and central events. Studies in adults have demonstrated that the progression from an individual respiratory event to a cyclic pattern of repetitive respiratory events is induced by a combination of anatomical and neurochemical factors in the control of respiratory drive and effects on the upper airway and chest wall musculature (20).

Previous studies in adults and children, have only investigated the cardiovascular response to isolated respiratory events $(8,10,11,14,21)$ and the effect of repetitive events has not been investigated. Therefore, the aims of this study were firstly to quantify the number of repetitive compared to isolated respiratory events and secondly to compare the HR and $\mathrm{BP}$ responses at event termination between these, in preschool children with SDB.

\section{RESULTS}

Demographic, sleep, and respiratory data for the two groups of children who exhibited just isolated events and those that exhibited both isolated and repetitive events are presented in

${ }^{1}$ The Ritchie Centre, Hudson Institute of Medical Research, Melbourne, Australia; ${ }^{2}$ Department of Paediatrics, Monash University, Melbourne, Australia; ${ }^{3}$ Melbourne Children's Sleep Centre, Monash Children's Hospital, Melbourne, Australia; ${ }^{4}$ Clinical Sciences Research, Murdoch Children's Research Institute, Melbourne, Australia. Correspondence: Lisa M. Walter (lisa.walter@hudson.org.au) 
Table 1. Twenty-eight children (62\%) exhibited both isolated events and episodes of repetitive respiratory events and 17 children exhibited only isolated respiratory events. There were no differences in gender, age or BMI z-score between the groups. The children who exhibited repetitive respiratory events had a significantly higher respiratory disturbance index $(P<0.001)$, obstructive apnea hypopnea index $(P<0.001)$, central apnea hypopnea index $(P<0.05)$, and arousal index $(P<0.05)$ compared with those who had isolated events only. There were no differences for the other sleep and respiratory parameters, or for the mean HR and pulse transit time (PTT) during wake or any of the sleep stages.

\section{Characteristics of Episodes of Repetitive Respiratory Events}

Central apneas were the predominate type of event within repetitive episodes, followed by hypopneas and respiratory event-related arousals (RERAs) (Figure 1a). Repetitive episodes most often began with a hypopnea (Figure $\mathbf{1 b}$ ) and the majority of repetitive episodes commenced during N1 sleep, followed by rapid eye movement (REM) (Figure 1c).

Data for the 28 children who exhibited both episodes of repetitive respiratory events and isolated events are presented in Table 2. A ratio of the number of events within an episode of repetitive respiratory events to the number of events that were isolated throughout the night for each of the 28 subjects was calculated. Eight children (29\%) had a repetitive events/isolated events ratio of less than 1.0, indicating that they had more respiratory events that were isolated than events that were in repetitive episodes. One child had a ratio of 1.0, indicating an equal number of events that were isolated and in repetitive episodes. Nineteen children $(69 \%)$ had a ratio greater than 1.0 , indicating more events that were in repetitive episodes/child than were isolated events/child.

Table 1. Demographic, sleep, and respiratory data for the whole cohort (i.e., children who exhibited episodes of repetitive respiratory events and those who had isolated events only)

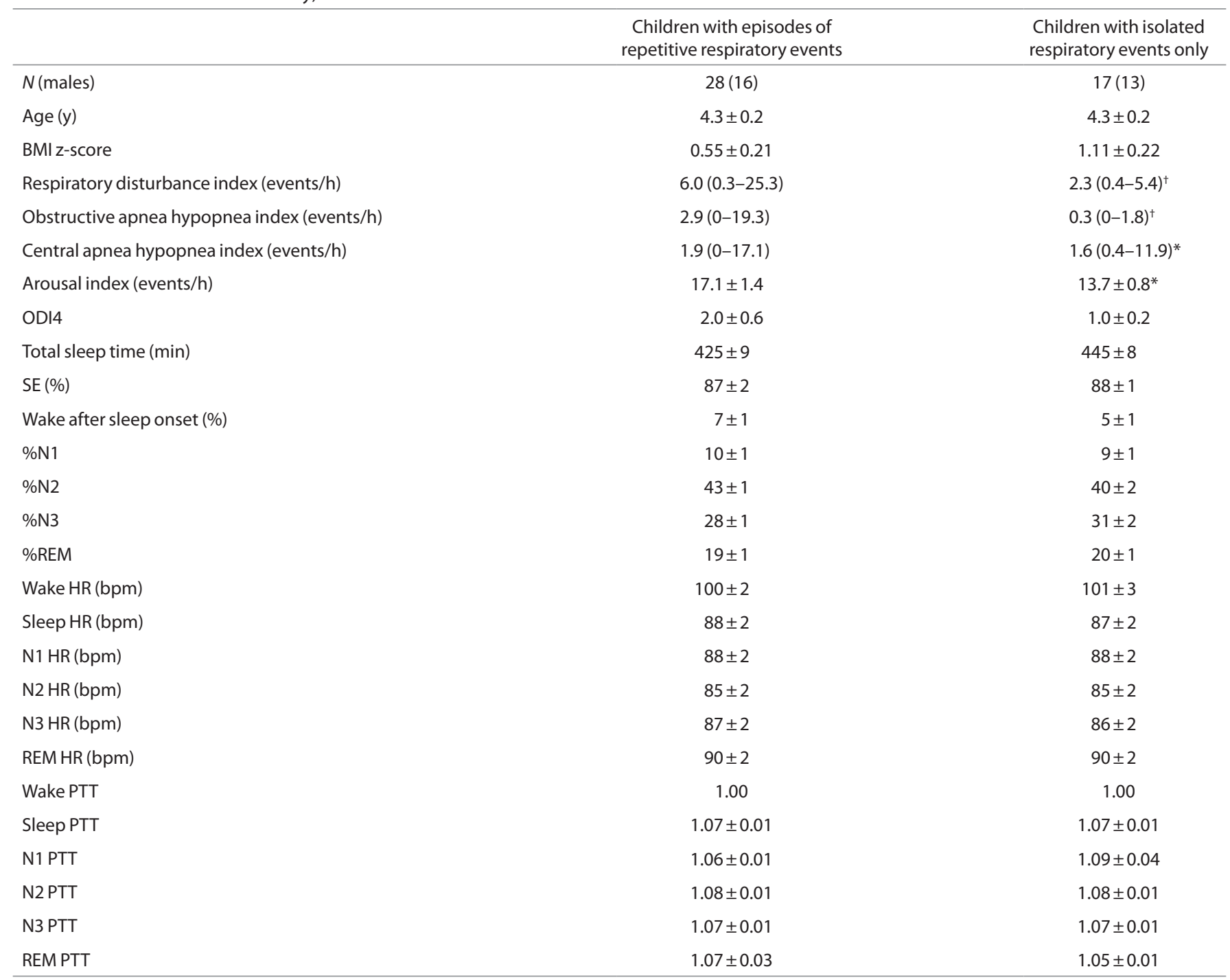

Data presented as mean \pm SEM or median (range).

${ }^{*} P<0.05 ;{ }^{\dagger} P<0.001$.

$\mathrm{HR}$, heart rate; $\mathrm{PTT}$, pulse transit time. 


\section{Cardiovascular Consequences of Repetitive vs. Isolated Respiratory Events}

For the episodes of repetitive events, the \% change in HR from late to between events and from late event to postepisode were significantly higher when compared with the \% change in HR from late to postevent in isolated respiratory events $(P<0.001$ for both), as shown in Figure 2a. The \% change in PTT from late event to postepisode was significantly greater (indicating a greater change in BP) for repetitive episodes when compared with the $\%$ change from late to postevents in isolated respiratory events $(P<0.05)$ (Figure $2 \mathbf{b})$. There was no difference between \% change in PTT from late to between events in repetitive episodes compared with \% change from late to postevents in isolated respiratory events.

\section{The Relationship Between Mean HR and PTT, and the Ratio of the Number of Repetitive Respiratory Events to the Number of Isolated Events Per Child During the Sleep Stages}

The ratio of repetitive to isolated respiratory events for each child significantly predicted the mean HR, which increased with an increasing proportion of repetitive respiratory events compared with isolated events during N1, N2, REM, and total sleep (Figure 3). There were no relationships between mean PTT and the ratio of repetitive to isolated respiratory events during wake or any sleep stage.

\section{Determinants of Percentage Change in HR and PTT From Late- to Between-Event Phases in Episodes of Repetitive Respiratory Events}

Results of the multiple linear regression analysis indicate that during total sleep, the repetitive to isolated events ratio, the number of individual respiratory events per episode, the number of arousals per episode, the ratio of the duration of the respiratory event to the duration of the subsequent between event period, and sleep stage were significantly, if weakly predictive of the \% change in HR from the late event phase to between the respiratory events (Table 3 ). The model predicted $44 \%$ of the $\%$ change in HR from the late event phase to between the respiratory events $(P<0.01)$. This suggests that the more repetitive
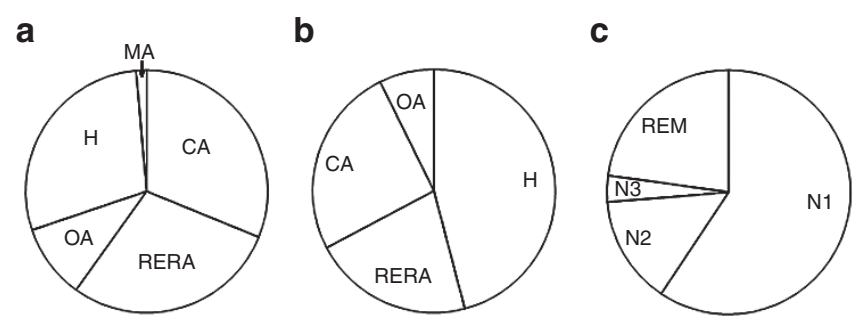

Figure 1. Pie charts representing data from children who exhibited repetitive respiratory events. (a) the types of respiratory events that made up the episodes of repetitive respiratory events: $H$, hypopneas (264 (29\%)); MA, mixed apneas (13 (1\%)); CA, central apneas (286 (31\%)); RERA, respiratory event related arousals (264 (29\%)); OA, obstructive apneas (91 (10\%)). (b) the types of respiratory events that initiated the episodes: OA $(25,(8 \%))$; $\mathrm{H}(157(46 \%)) ;$ RERA (73 (21\%)); CA (87 (25\%)). (c) The sleep stage in which the episodes commenced: N1 (203 (59\%)); N2 (49 (14\%)); N3 (12 (4\%)); $\operatorname{REM}(78(23 \%))$. events compared with isolated events, the more episodes, the more events and arousals per episode a child has, the higher the surge in HR. In contrast, there is a negative relationship between the surge in HR and sleep stage, suggesting that the HR surge is highest during N1 and decreases during N2, N3, and REM. There is also a weak negative relationship between the surge in HR and the ratio of the duration of an event to the duration of the between event period, suggesting that the closer together the events become the \% change in HR is not as great.

Further analyses separating the repetitive respiratory event episodes into the sleep stage in which they occurred demonstrated that when the episodes occurred during N1 sleep, the predictive variables remained the same as for total sleep. When the episodes occurred during N2 or REM sleep, only the number of arousals in the episode predicted the change in $\operatorname{HR}\left(r^{2}=0.3, P<0.01\right.$; REM: $\left.r^{2}=0.5, P<0.001\right)$. None of the variables were predictive of the late- to between-events percentage change in PTT during any sleep stage.

\section{DISCUSSION}

$\mathrm{SDB}$ is common in preschool aged children and can have significant effects on the cardiovascular system, which are thought to be a consequence of the repeated surges in HR and $\mathrm{BP}$ that occur at event termination. In this study, we demonstrate for the first time that episodes of repetitive respiratory events predominate in children and furthermore, are

Table 2. Episodes of repetitive respiratory events and isolated respiratory events in the 28 children who exhibited both

\begin{tabular}{lc}
\hline Respiratory events and responses & Number \\
\hline Total number of repetitive episodes analyzed & 342 \\
Number of repetitive episodes per child & $12(1-35)$ \\
Total number of individual events analyzed & 918 \\
Number of events per episode & $26(2-59)$ \\
Repetitive episode duration (s) & $90(17-1022)$ \\
Individual repetitive event duration (s) & $13(5-68)$ \\
Number of isolated events per child & $21(3-55)$ \\
Isolated event duration (s) & $10(4-46)$ \\
Repetitive events/isolated events per child & $1.73(0.16-17)$ \\
Repetitive events & \\
$\%$ change in HR from late to between events & $27 \pm 1^{\dagger}$ \\
$\%$ change in HR from late event to postepisode & $25 \pm 1^{\dagger}$ \\
$\%$ change in PTT from late to between events & $-3 \pm 0.2$ \\
$\%$ change in PTT from late event to postepisode & $-8 \pm 2^{*}$ \\
Isolated events & \\
$\%$ change in HR from late to postevent & $17 \pm 1$ \\
\% change in PTT from late to postevent & $-2 \pm 2$ \\
\hline
\end{tabular}

Results are presented as median (range) or mean \pm SEM.

${ }^{*} P<0.05$ compared with $\%$ change in PTT from late to postevent. ${ }^{+} P<0.001$ compared with isolated events \% change in HR from late to postevent.

$H R$, heart rate; PTT, pulse transit time. 
associated with a greater cardiovascular effect than isolated events. We have identified that the surges in HR that occur between successive events within an episode of repetitive events, and at the termination of the episodes, are 8 to $10 \%$ higher compared to that following isolated events in the same children. Although not as pronounced, the concurrent changes in BP, indicated by decreased PTT, were also higher. In addition, the mean HR during N1, N2, N3, REM, and total sleep increased with an increasing proportion of repetitive respiratory events compared with isolated events. Our study suggests that it is not only the total number of respiratory events that a child has that is of importance, but also whether they form a repetitive cycle of events, potentiating an accentuated cardiovascular effect.
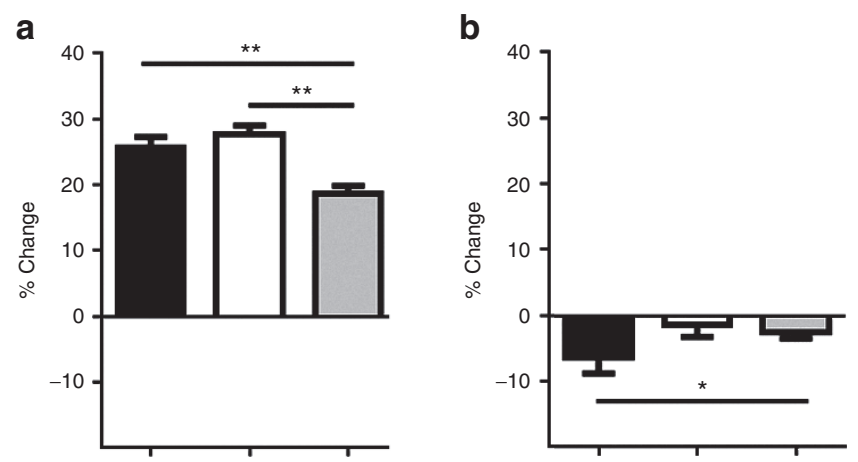

Figure 2. Percentage change in heart rate (a) and pulse transit time (b) from the late- to postepisode (black columns) and late-to between-events (white columns) for episodes of repetitive respiratory events compared with late- to postevent for isolated respiratory events (gray columns). Data presented as mean \pm SEM. ${ }^{*} P<0.05 ;{ }^{* *} P<0.001$.
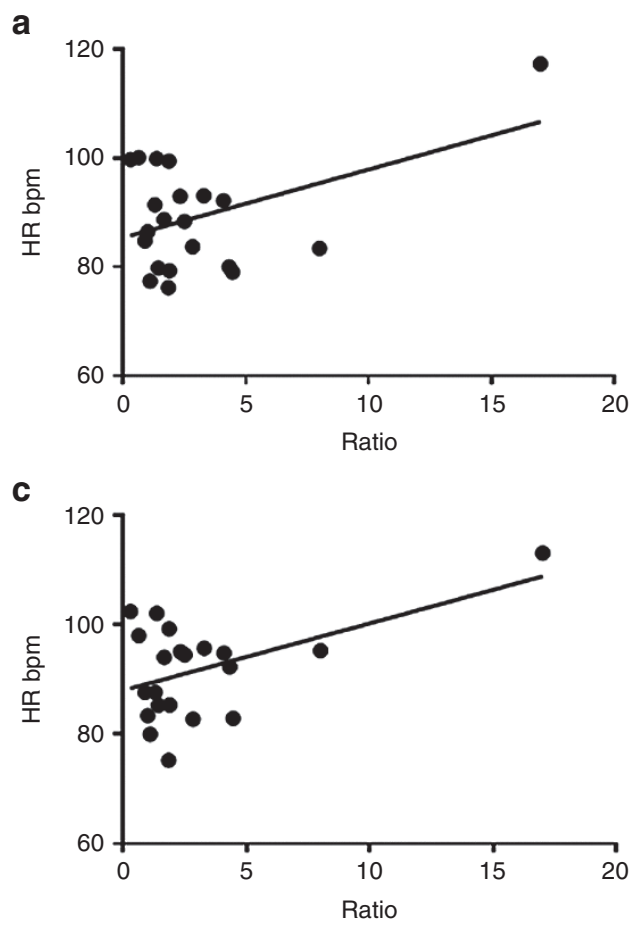

The mean \% change in HR between late-events and successive between-event phases (27\%) and also between late-events and the 15 s period following an episode (25\%) is significantly higher compared with the late to postevent phase changes that occur following isolated events (17\%). This result for isolated respiratory events is similar in magnitude to the surge in HR reported in previous analyses of only isolated events (11). Also similar to the previous study, the surge in BP at the termination of an event was much smaller compared with HR. Previous studies have identified that both isolated obstructive and central events in children have similar cardiovascular sequelae to isolated obstructive events in adults $(10,14)$. The episodes of repetitive respiratory events included in this study, included substantial numbers of central events. These episodes can last for up to $5 \mathrm{~min}$, with some children having numerous such episodes during the night. With a further 8 to $10 \%$ increase in the HR surge associated with repetitive respiratory events, which comprise the majority of events, further research is needed to evaluate the effect this has on the cardiovascular health of such young children

It is a common belief that the adverse cardiovascular effects of SDB are in part due to the intermittent hypoxia that characterizes the condition. Rodent models of long-term intermittent hypoxia have demonstrated hypertension and increased sympathetic activity (22), increased right ventricular systolic pressure, right ventricular mass, neovascularization of distal pulmonary vessels (23), systemic and pulmonary vascular pressure $(24,25)$, cardiac hypertrophy, and cardiac and perivascular fibrosis (26). Previous studies in children have however not found a strong link between arterial desaturation and elevated HR and BP (27). Similarly in our study, oxygen desaturation

b

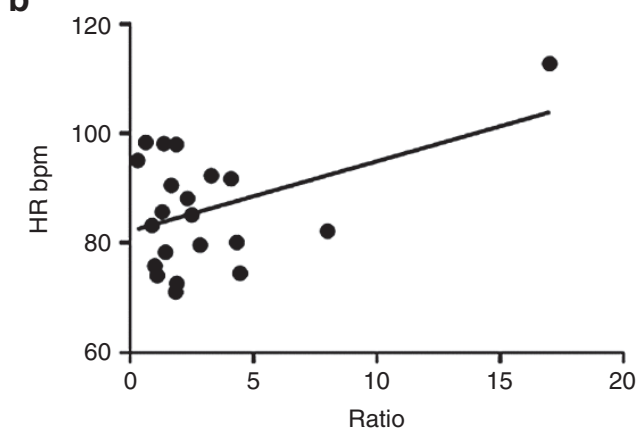

d

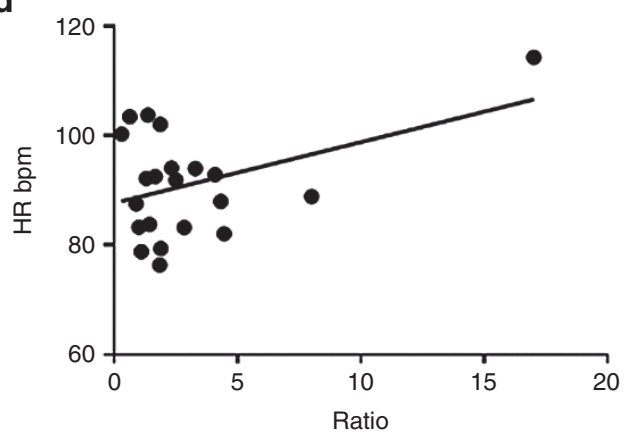

Figure 3. The relationship between mean heart rate and the ratio of the number of repetitive respiratory events to the number of isolated events per child during (a) N1, $R^{2}=0.2 ; P=0.04$. (b) $\mathrm{N} 2, R^{2}=0.1 ; P=0.04$. (c) $\mathrm{REM}, R^{2}=0.2 ; P=0.02$ and (d) total sleep, $R^{2}=0.2 ; P=0.04$. 
Table 3. Significant determinants of percentage change in heart rate from late- to between-event phases during total sleep identified with multiple linear regression analysis

\begin{tabular}{lccc}
\hline & $r^{2}$ & $\operatorname{Std} \beta$ & $P$ \\
\hline $\begin{array}{l}\text { Repetitive events/isolated events } \\
\text { per child }\end{array}$ & 0.04 & 0.1 & $<0.001$ \\
$\begin{array}{l}\text { Number of repetitive episodes per } \\
\text { child }\end{array}$ & 0.14 & 0.1 & 0.03 \\
$\begin{array}{l}\text { Number of arousals per episode } \\
\begin{array}{l}\text { Respiratory event duration/ } \\
\text { between event duration }\end{array}\end{array}$ & 0.16 & 0.3 & $<0.001$ \\
$\begin{array}{l}\text { Sleep stage } \\
\text { N }\end{array}$ & 0.20 & -0.2 & $<0.001$ \\
& & & $<0.1$ \\
\hline
\end{tabular}

during the episodes of repetitive respiratory events was not predictive of an increase in the HR or PTT surge between repetitive respiratory events, which suggests that hypoxia did not play an important role in the increased HR and PTT in these preschool-aged children.

Similarly, the effects of arousals on the cardiovascular system have been well documented, demonstrating that arousals are associated with increased HR (28). While there was a significantly higher arousal index in the children who had repetitive respiratory events compared with those who only had isolated events ( 17 vs. 14 events/h), this reflects that the children with only isolated events also had fewer respiratory events overall. Furthermore, the number of arousals within a repetitive respiratory event episode was significantly, if weakly predictive of the \% change in HR from the late event phase to between the respiratory events, suggesting that the more arousals during an episode, the higher the surge in HR.

Sleep stage was a significant determinant of the HR surge. The majority of episodes occurred during N1 stage sleep. This finding has also been reported in studies of repetitive respiratory events in adults with OSA (29). It has been hypothesized that at the transition from wake to sleep, several respiratory control mechanisms are down regulated, upper airway dilator and respiratory pump muscle tone is reduced, and there is a reduction in chemosensitivity leading to respiratory instability $(29,30)$. Furthermore, hypopneas can be induced when the transition to sleep occurs rapidly and there is a delay in the compensatory response from the chemoreceptors (31). In accordance with this theory, the majority of episodes in our study began with either a hypopnea or a RERA, and during N1 sleep.

In addition to the effects of hypoxia and arousals, the cardiovascular effects of SDB in adults have been attributed to a number of interrelated pathophysiological responses to respiratory events (32). Following obstructive apneas and hypopneas, there is an increase in sympathetic activity resulting in peripheral vasoconstriction and increased $\mathrm{HR}$, which persists during the daytime (33). It has been hypothesized that the increased transmural gradients across the atria, ventricles and aorta, which may disrupt ventricular function and result from changes in intrathoracic pressure during obstructive events, lead to autonomic and hemodynamic instability (32). OSA has been associated with impaired right and left ventricular function in adults (34), and similarly, children with OSA have been identified with left ventricular hypertrophy and cardiac remodeling $(6,7)$. Studies have not been conducted to determine the effect of repetitive changes in intrathoracic pressure when there are a large number of respiratory events that are occurring close together, on autonomic and hemodynamic instability. Our findings indicate greater hemodynamic instability during repetitive events in preschool-aged children compared with isolated events. However, we did not investigate autonomic function and future research is required to elucidate the effect of repetitive respiratory events on autonomic function.

We acknowledge that this study had limitations. Data from a relatively small number of children were analyzed for this study and confirmation of the findings in a larger cohort of children is needed. A further limitation of the study, was that PTT is a surrogate measure of BP change and therefore may not reflect true mean systolic BP values. Additionally, due to the invasive nature of its measurement, sympathetic activity at respiratory event termination was not able to be obtained. These would have complemented our data and increased our understanding of the cardiovascular sequelae to repetitive respiratory events in children.

\section{Conclusions}

This study has identified that respiratory events in preschool children with SDB most commonly occur in episodes of repetitive events, rather than isolated events. The cardiovascular sequelae of episodes of repetitive respiratory events are significantly more pronounced than those of isolated events. These findings highlight that the temporal nature of respiratory events should be taken into consideration when the clinical assessment of SDB severity is being made.

\section{METHODS}

Ethical approval for this project was granted by the Monash Health and Monash University Human Research Ethics Committees. Written informed consent was obtained from parents and verbal assent from the children after a full explanation of the procedure. There was no monetary incentive for participation.

\section{Subjects}

In the present study, we analyzed the overnight polysomnography (PSG) recordings of 45 children (3-5 y) diagnosed with SDB (obstructive apnea hypopnea index $(\mathrm{OAHI})>1$ ). This study represents baseline data for children who returned for a follow-up study investigating the effects of treatment on preschool children with SDB. Children who were born preterm, or with conditions or taking medications known to affect sleep, breathing, BP, or neurocognitive function were not recruited.

\section{Protocol}

At the time of the PSG, children were otherwise healthy and not undergoing treatment with either nasal steroids, leukotriene receptor antagonists or antibiotics. Prior to the PSG, height and weight were measured and converted to a BMI z-score to adjust for gender and age (35).

\section{Polysomnography}

Overnight PSG was performed according to a previously published protocol (11). In brief, electrophysiological signals were recorded 
using a commercially available PSG system (E-Series, Compumedics, Melbourne, Australia) and standard pediatric recording techniques (36). Electrodes for recording electroencephalogram, left and right electrooculogram, submental electromyogram, left and right anterior tibialis muscle electromyogram, and electrocardiogram were attached. Other recordings included thoracic and abdominal breathing movements, transcutaneous carbon dioxide, nasal pressure, oronasal airflow, and oxygen saturation $\left(\mathrm{SpO}_{2}\right)$. A photoplethysmographic pulse oximeter (PPG) (Adult Flex Sensor 3M, Nonin Medical, Plymouth, MN) was used to record the pulse waveform necessary for PTT analysis.

\section{Polysomnographic Analysis}

PSG studies were manually sleep-staged into $30 \mathrm{~s}$ epochs and respiratory events scored by experienced pediatric sleep technologists according to slightly modified AASM 2007 rules that were used clinically $(36,37)$. Modification consisted of the inclusion of RERA associated with arousal or desaturation into the OAHI. As the AASM 2007 rules resulted in a lower OAHI and lesser OSA severity compared with the previous rules used in our laboratory, inclusion of RERAs leads to a comparable OAHI to the updated AASM rules in 2012 (37). RERAs were scored where there was a discernible decrease in amplitude and flattening of the nasal pressure trace, associated with snoring, noisy breathing, elevation of the end-tidal or transcutaneous $\mathrm{pCO}_{2}$ and/or visual evidence of increased work of breathing, leading to an arousal from sleep and/or $\geq 3 \%$ desaturation.

Variables calculated from the PSG according to previously published definitions $(5,11,38)$, were the respiratory disturbance index, OAHI, central apnea hypopnea index, wake after sleep onset, total sleep time, arousal index, and the number of times the $\mathrm{SpO}_{2}$ dropped by $\geq 4 \%$ per h total sleep time (ODI4).

An episode of repetitive respiratory events was defined as two or more respiratory events that occurred within $25 \mathrm{~s}$ of the preceding event. As there has been no previous definition this time was chosen as it was considered the minimum time allowable between isolated events in previous studies $(10,11)$. The episodes of repetitive respiratory events included both obstructive and central events, as previous studies in children have identified similar HR and BP responses to isolated obstructive and central events $(10,14)$.

\section{Data Analysis}

All PSG data were transferred via European Data Format to specialized data analysis software (LabChart 7.2, ADInstruments, Sydney, Australia). Thirty second epochs containing wake after sleep onset were excluded, together with epochs containing manually identified movement artifact including gross body movements affecting all PSG channels or artifact isolated to electrocardiogram or PPG signals. PTT and HR data for total sleep were then grouped according to sleep stage (Wake, N1, N2, N3, REM) and a mean value for each subject calculated. We examined the types of events which made up the repetitive respiratory episodes (obstructive apneas, hypopneas, RERAs, central apneas, and mixed apneas) and the sleep stages in which they occurred.

\section{PTT Analysis}

PTT was calculated as a surrogate measure of BP change, using a previously published method (5). This has been shown to have good agreement with direct measurements of BP change using photoplethysmography (39). The peak of the electrocardiogram R-wave and the $50 \%$ point of the height of the corresponding pulse wave, were identified by peak detection for each beat in LabChart. PTT was calculated as the time delay between those two points. An average value was calculated for each $30 \mathrm{~s}$ epoch during sleep, which was normalized to that child's mean PTT during restful wake prior to sleep onset.

\section{Beat-By-Beat PTT and HR Analysis}

To compare the effects of repetitive respiratory events compared to isolated events the data for the 28 children who exhibited both episodes of repetitive respiratory events and isolated events were examined. Beat-by-beat HR and PTT were analyzed as previously described (11). Events were averaged for two phases during each apnea/hypopnea and were termed early-event (first half) and lateevent (second half) $(10,11,14)$. Division of the event as such allowed for comparison of data between events of differing durations. The characteristics associated with each event were recorded, including sleep stage (N1, N2, N3, and REM) in which it occurred, arousal (arousal or no arousal), event type (obstructive apnea, hypopnea or RERA, central apnea, mixed apnea), and oxygen desaturation $>4 \%$ (desaturation, no desaturation) and event length. Prior to each episode of repetitive respiratory events, a $10 \mathrm{~s}$ baseline of stable HR and PTT was identified within the same sleep stage as the event. Within each episode of repetitive events, each individual respiratory event was divided into early- and late-phase and the mean of the three consecutive beats at HR peak or PTT trough for the period between consecutive events was calculated. This was chosen as previous studies in children have determined that the most significant percentage change in HR and BP occurred between the late- and postevent phases of isolated respiratory events $(10,11,14)$. Following the final respiratory event of the episode, the postepisode phase was identified as being the $15 \mathrm{~s}$ following the last event and the mean of the three consecutive beats at HR peak or PTT trough for this phase was calculated. A similar technique was used for isolated events.

\section{Statistical Analyses}

Statistical analysis was performed using SPSS (version 20.0, IL). Data were first tested for normality and equal variance. For the whole cohort, demographic, sleep, and respiratory parameters were compared between the children who exhibited episodes of repetitive respiratory events and those who exhibited isolated events only, using Mann-Whitney $U$-tests. In the group of 28 children who exhibited both isolated and repetitive events, the \% change in HR and PTT from the late event phase to between successive events and from the late event phase to the postepisode phase in episodes of repetitive events, was compared with the late to postevent phase in isolated events using Student's $t$-tests. A ratio of the number of events within an episode of repetitive respiratory events to the number of events that were isolated throughout the night for each subject was calculated and linear regression was used to determine whether this ratio predicted changes in mean HR and PTT for each subject during wake, N1, N2, N3, REM, and total sleep.

To determine the factors affecting the late-to-between events \% change in HR and PTT, forward stepwise multiple linear regression was performed using the following independent variables: the number of episodes of repetitive respiratory events, the ratio of repetitive to isolated respiratory events, the duration of the episodes, the duration of the events within the episode, the number of arousals associated with events within the episode, the number of oxygen desaturations $\geq$ $4 \%$ associated with events within the episode, sleep stage and the ratio of event duration to the subsequent between event duration. $P \leq 0.05$ was considered significant.

\section{ACKNOWLEDGMENT}

The authors would like to thank the children and families who participated in this study and the staff of the Melbourne Children's Sleep Centre for their support.

\section{STATEMENT OF FINANCIAL SUPPORT}

This study was supported by a National Health and Medical Research Council of Australia project grant 1008919 and the Victorian Government's Operational Infrastructure Support Program.

Disclosures: The authors have no other financial support or conflicts of interest to disclose.

\section{REFERENCES}

1. Mindell JA, Owens JA. Pediatric Sleep, Diagnosis and Management of Sleep Problems. 2nd edn. Philadelphia: Lippincott Williams and Wilkins, 2010.

2. Mindell JA, Owens JA. Sleep-disordered breathing and obstructive sleep apnea syndrome. Pediatric Sleep: Diagnosis and Management of Sleep Problems. 2nd edn. Philadelphia: Lippincott Williams and Wilkins, a Wolters Kluwer Health business, 2010. pp. 100-15. 
3. Horne RS, Yang JS, Walter LM, et al. Elevated blood pressure during sleep and wake in children with sleep-disordered breathing. Pediatrics 2011;128:e85-92.

4. Li AM, Au CT, Sung RY, et al. Ambulatory blood pressure in children with obstructive sleep apnoea: a community based study. Thorax 2008;63: 803-9.

5. Nisbet LC, Yiallourou SR, Biggs SN, et al. Preschool children with obstructive sleep apnea: the beginnings of elevated blood pressure? Sleep 2013;36:1219-26.

6. Amin R, Somers VK, McConnell K, et al. Activity-adjusted 24-hour ambulatory blood pressure and cardiac remodeling in children with sleep disordered breathing. Hypertension 2008;51:84-91.

7. Amin RS, Kimball TR, Bean JA, et al. Left ventricular hypertrophy and abnormal ventricular geometry in children and adolescents with obstructive sleep apnea. Am J Respir Crit Care Med 2002;165:1395-9.

8. Hamilton GS, Solin P, Naughton MT. Obstructive sleep apnoea and cardiovascular disease. Intern Med J 2004;34:420-6.

9. Stoohs R, Guilleminault C. Cardiovascular changes associated with obstructive sleep apnea syndrome. J Appl Physiol (1985) 1992;72:583-9.

10. O'Driscoll DM, Foster AM, Ng ML, et al. Acute cardiovascular changes with obstructive events in children with sleep disordered breathing. Sleep 2009;32:1265-71.

11. Nisbet LC, Yiallourou SR, Nixon GM, et al. Characterization of the acute pulse transit time response to obstructive apneas and hypopneas in preschool children with sleep-disordered breathing. Sleep Med 2013;14: 1123-31.

12. Traeger N, Schultz B, Pollock AN, Mason T, Marcus CL, Arens R. Polysomnographic values in children 2-9 years old: additional data and review of the literature. Pediatr Pulmonol 2005;40:22-30.

13. Montgomery-Downs HE, O’Brien LM, Gulliver TE, Gozal D. Polysomnographic characteristics in normal preschool and early school-aged children. Pediatrics 2006;117:741-53.

14. O'Driscoll DM, Foster AM, Ng ML, et al. Central apnoeas have significant effects on blood pressure and heart rate in children. J Sleep Res 2009;18:415-21.

15. Okabe S, Hida W, Kikuchi Y, et al. Role of hypoxia on increased blood pressure in patients with obstructive sleep apnoea. Thorax 1995;50:28-34.

16. Ringler J, Basner RC, Shannon R, et al. Hypoxemia alone does not explain blood pressure elevations after obstructive apneas. J Appl Physiol (1985) 1990;69:2143-8.

17. Azarbarzin A, Ostrowski M, Hanly P, Younes M. Relationship between arousal intensity and heart rate response to arousal. Sleep 2014;37:645-53.

18. Bonnet MH, Arand DL. Heart rate variability: sleep stage, time of night, and arousal influences. Electroencephalogr Clin Neurophysiol 1997;102:390-6.

19. Somers VK, White DP, Amin R, et al.; American Heart Association Council for High Blood Pressure Research Professional Education Committee, Council on Clinical Cardiology; American Heart Association Stroke Council; American Heart Association Council on Cardiovascular Nursing; American College of Cardiology Foundation. Sleep apnea and cardiovascular disease: an American Heart Association/american College Of Cardiology Foundation Scientific Statement from the American Heart Association Council for High Blood Pressure Research Professional Education Committee, Council on Clinical Cardiology, Stroke Council, and Council On Cardiovascular Nursing. In collaboration with the National Heart, Lung, and Blood Institute National Center on Sleep Disorders Research (National Institutes of Health). Circulation 2008;118:1080-111.
20. Dempsey JA, Veasey SC, Morgan BJ, O’Donnell CP. Pathophysiology of sleep apnea. Physiol Rev 2010;90:47-112.

21. Imadojemu VA, Gleeson K, Gray KS, Sinoway LI, Leuenberger UA. Obstructive apnea during sleep is associated with peripheral vasoconstriction. Am J Respir Crit Care Med 2002;165:61-6.

22. Lucking EF, O'Halloran KD, Jones JF. Increased cardiac output contributes to the development of chronic intermittent hypoxia-induced hypertension. Exp Physiol 2014;99:1312-24.

23. Fagan KA. Selected Contribution: Pulmonary hypertension in mice following intermittent hypoxia. J Appl Physiol (1985) 2001;90:2502-7.

24. Campen MJ, Shimoda LA, O’Donnell CP. Acute and chronic cardiovascular effects of intermittent hypoxia in C57BL/6J mice. J Appl Physiol (1985) 2005;99:2028-35.

25. Nisbet RE, Graves AS, Kleinhenz DJ, et al. The role of NADPH oxidase in chronic intermittent hypoxia-induced pulmonary hypertension in mice. Am J Respir Cell Mol Biol 2009;40:601-9.

26. Nishioka S, Yoshioka T, Nomura A, et al. Celiprolol reduces oxidative stress and attenuates left ventricular remodeling induced by hypoxic stress in mice. Hypertens Res 2013;36:934-9.

27. Gozal D, Hakim F, Kheirandish-Gozal L. Chemoreceptors, baroreceptors, and autonomic deregulation in children with obstructive sleep apnea. Respir Physiol Neurobiol 2013;185:177-85.

28. Trinder J, Allen N, Kleiman J, et al. On the nature of cardiovascular activation at an arousal from sleep. Sleep 2003;26:543-51.

29. Eckert DJ, Malhotra A, Jordan AS. Mechanisms of apnea. Prog Cardiovasc Dis 2009;51:313-23.

30. Dunai J, Wilkinson M, Trinder J. Interaction of chemical and state effects on ventilation during sleep onset. J Appl Physiol (1985) 1996;81:2235-43.

31. Khoo MC, Gottschalk A, Pack AI. Sleep-induced periodic breathing and apnea: a theoretical study. J Appl Physiol (1985) 1991;70:2014-24.

32. Kuniyoshi FH, Pusalavidyasagar S, Singh P, Somers VK. Cardiovascular consequences of obstructive sleep apnoea. Indian J Med Res 2010;131: 196-205.

33. Narkiewicz K, Montano N, Cogliati C, van de Borne PJ, Dyken ME, Somers VK. Altered cardiovascular variability in obstructive sleep apnea. Circulation 1998;98:1071-7.

34. Romero-Corral A, Somers VK, Pellikka PA, et al. Decreased right and left ventricular myocardial performance in obstructive sleep apnea. Chest 2007;132:1863-70.

35. Ogden CL, Kuczmarski RJ, Flegal KM, et al. Centers for Disease Control and Prevention 2000 growth charts for the United States: improvements to the 1977 National Center for Health Statistics version. Pediatrics 2002;109:45-60.

36. Iber C, Ancoli-Israel S, Chesson A, Quan SF. The AASM Manual for the Scoring of Sleep and Associated Events: Rules, Terminology and Technical Specification. 1st edn. Westchester, IL: American Academy of Sleep Medicine, 2007.

37. Nixon GM, Hyde M, Biggs SN, Walter LM, Horne RS, Davey MJ. The impact of recent changes to the respiratory scoring rules in pediatrics. J Clin Sleep Med 2014;10:1217-21.

38. Nisbet LC, Yiallourou SR, Nixon GM, et al. Nocturnal autonomic function in preschool children with sleep-disordered breathing. Sleep Med 2013;14:1310-6.

39. Vlahandonis A, Biggs SN, Nixon GM, Davey MJ, Walter LM, Horne RS. Pulse transit time as a surrogate measure of changes in systolic arterial pressure in children during sleep. J Sleep Res 2014;23:406-13. 